\title{
Optimal Combination of Base Station Densities for Energy-Efficient Two-Tier Heterogeneous Cellular Networks
}

\author{
Dongxu Cao, Student Member, IEEE, Sheng Zhou, Member, IEEE, and Zhisheng Niu, Fellow, IEEE
}

\begin{abstract}
In this paper, the optimal BS (Base Station) density for both homogeneous and heterogeneous cellular networks to minimize network energy cost is analyzed with stochastic geometry theory. For homogeneous cellular networks, both upper and lower bounds of the optimal BS density are derived. For heterogeneous cellular networks, our analysis reveals the best type of BSs to be deployed for capacity extension, or to be switched off for energy saving. Specifically, if the ratio between the micro BS cost and the macro BS cost is lower than a threshold, which is a function of path loss and their transmit power, then the optimal strategy is to deploy micro BSs for capacity extension or to switch off macro BSs (if possible) for energy saving with higher priority. Otherwise, the optimal strategy is the opposite. The optimal combination of macro and micro BS densities can be calculated numerically through our analysis, or alternatively be conservatively approximated with a closed-form solution. Based on the parameters from EARTH, numerical results show that in the dense urban scenario, compared to the traditional macro-only homogeneous cellular network with no BS sleeping, deploying micro BSs can reduce about $40 \%$ of the total energy cost, and further reduce up to $35 \%$ with BS sleeping capability.
\end{abstract}

Index Terms-Base station density, Poisson point process, energy efficiency, heterogeneous cellular networks.

\section{INTRODUCTION}

W ITH the rapid increase of mobile subscribers as well as the traffic demand, cellular networks have experienced several significant evolutions, from the first generation to the current CDMA-based 3G cellular networks. To handle the ever growing traffic requirement and minimize the network cost, both LTE [2] and WiMAX [3] standard groups have proposed the "micro" BS (Base Station) concept, such as femtocell [4][5] and picocell [6], which will bring heterogeneousness into cellular networks [7][8].

By doing so, how to plan heterogeneous cellular networks becomes an important issue, especially for capacity extension through deploying more BSs based on existing networks. It

Manuscript received August 24, 2012; revised January 30 and May 17, 2013; accepted June 19, 2013. The associate editor coordinating the review of this paper and approving it for publication was D. Tarchi.

The authors are with Tsinghua National Laboratory for Information Science and Technology, Dept. of Electronic Engineering, Tsinghua University, Beijing 100084, China (e-mail: cdx08@mails.tsinghua.edu.cn, \{sheng.zhou, niuzhs\}@tsinghua.edu.cn).

Part of the work has been presented in ICC 2012 [1]. This work is sponsored in part by the National Basic Research Program of China (2012CB316001), the Nature Science Foundation of China (61201191, 60925002, 61021001), and Hitachi Ltd.

Digital Object Identifier 10.1109/TWC.2013.080113.121280 is different from the traditional cellular network planning. Because as Qualcomm in the white paper 2011 [7] has mentioned, the deployment of micro BSs may be more or less ad hoc, based on just a rough knowledge of coverage issues and traffic density in the network.

On the other hand, today's cellular networks are consuming much energy. Taking China as an example, there had been over 1.0 million BSs deployed by the three major operators consuming as much as 28.9 billion KWh electric power a year by 2009 [9]. In the future, due to the exponential increase of wireless data traffic, energy may be another limit factor for cellular networks. Accordingly, recent research demonstrated that a system-wide approach by adapting the BS density through turning on/off BSs to the actual traffic load is effective [10]-[12].

In this paper, in order to assist the capacity extension and dynamic BS switching for heterogeneous cellular networks, we focus on the BS density problem: given the predefined QoS requirement and traffic load, what is the optimal BS density to minimize the network cost? For network planning, even if an answer to this problem cannot give us the precise location of each BS, it can provide valuable information about the type and the number of BSs required. For energy saving, the answer shows what type of and how many BSs can be switched off when the traffic load is low.

\section{A. Related Work}

Recently, energy saving issue in cellular networks has become increasingly important. Accordingly, dynamic operation control schemes to optimize energy efficiency in cellular networks have been proposed [10]-[12], which mainly focus on dynamic BS sleeping. There are also many works [13]-[16] on heterogeneous cellular networks to improve energy efficiency without BS sleeping, showing the benefits of low power micro BS sites through system-level simulations. Nevertheless, few of the above focus on BS density, or jointly consider dynamic BS sleeping in heterogeneous cellular networks.

Current research on cellular network planning mainly focus on designing practical deployment algorithm [17]-[22]. Mostly, it is an NP-hard mixed integer programming problem with many system parameters and constraints, and thus many numerical solutions have been proposed, e.g., genetic algorithms [18][19], tabu search [20][21], and Lagrange relaxation [22]. However, each solution is problem-specific and can be viewed as the subsequent step of our BS density problem. 
Nevertheless, among the very limited work on BS density, Ref. [23] is particularly related, since it attempts to find out the maximal inter-BS distance for CDMA cellular networks based on the hexagonal cellular network model. In [23], a simple rule is obtained, showing that the optimal inter-BS distance $d^{*}$ is proportional to the inverse of the square root of the user density $\lambda^{-\frac{1}{2}}$. However, this work cannot be used for capacity extension or dynamic BS sleeping, and is difficult to extend to heterogeneous cellular networks.

In this paper, we assume the location of BSs, either the macro or the micro, follows a random point process, PPP (Poisson Point Process) [24]-[27], and the cell topology is determined as the Voronoi tessellation [28]-[30] (weightedVoronoi tessellation for heterogeneous networks), in which users are always associated with the BS with the strongest mean received signal. The use of stochastic geometry for the analysis and design of wireless networks is surveyed in [25].

Particularly, our work is mainly based on the results from [26]. In [26], a new homogeneous cellular network model for multi-cell SINR (signal to interference and noise ratio) using stochastic geometry is developed, and the user coverage probability is obtained. Our work extends [26] to optimal BS density design for cellular network planning and dynamic BS sleeping. Moreover, heterogeneous cellular networks are also considered. Recently, the authors of [26] also derive the coverage probability for multi-tier heterogeneous networks in [27], and show that adding more tiers and/or BSs neither increases nor decreases the coverage probability for interferencelimited open access networks. Different from [27] on the signal level analysis, we additionally consider more features such as random cell size, available spectrum and capacity requirement to get the most cost-efficient combination of BS densities in two-tier heterogeneous networks.

\section{B. Contributions and Organization}

In this paper, we focus on the optimal BS density problem for both homogeneous and heterogeneous cellular networks with the service outage probability constraint. For homogeneous cellular networks, the optimal BS density can be achieved numerically. To exploit the impact of system parameters, we have derived the upper and lower bounds of the optimal BS density, and our numerical results show that the upper and lower bounds are both tight. Moreover, the upper bound is relaxed to have a closed-form formula.

For two-tier heterogeneous cellular networks, the cell topology is a weighted-Voronoi tessellation. However, to our best knowledge, there is no explicit analytical expression of the cell size distribution. Thus, first we do a numerical fitting of the macro and micro cell size distribution. We further verify that the cell size distribution is accurately predicted by Gamma functions.

Then, we solve the following two problems for heterogeneous cellular networks. (i) Capacity extension: if there have already been deployed with some macro and micro BSs, then which type of BSs is required and what is the optimal BS density to extend network capacity? (ii) Energy saving: when the traffic intensity is low, how many macro BSs and/or micro BSs should be switched off? These two problems are generalized into a uniform optimal BS density combination problem, and a simple rule is derived: If the cost per micro BS is lower than a threshold, then micro BSs are preferable. Otherwise, macro BSs are perferable. The optimal BS density can be calculated numerically through our analysis, and the closed-form conservative approximate solution is also given.

The rest of this paper is organized as follows: In Section II, the homogeneous and heterogeneous cellular system models are presented. Then the optimal BS density for the homogeneous cellular model is analyzed in Section III, after which the heterogeneous cellular network scenario is considered in Section IV. Finally, Section V concludes this paper.

\section{Cellular System Model}

Both homogeneous and heterogeneous cellular networks are considered in this paper. In homogeneous cellular networks, only one type of BSs are deployed in the systems, while heterogeneous cellular networks consist of two types of BSs: macro BSs with high transmit power and high unit cost, and micro BSs with low transmit power and low unit cost. To make the analysis tractable, we propose the following assumptions.

Assumption 1. BS and user locations: In both homogeneous and heterogeneous networks, all the BSs and the users are located in the infinite Euclidean plane according to independent homogeneous PPPs.

There are several reasons for choosing this BS location model: I) Though the PPP model is not an exact fit, it reasonably approximates the variability introduced by practical constraints of macro BS locations [31] and the potential random deployment of micro BSs [7]. II) This model is suitable to analyze capacity extension through deploying more BSs, because the superposition of two or more independent PPPs is still a PPP [25]. III) This model is also suitable to analyze energy saving through dynamic BS sleeping, because the independent thinning of a PPP is still a PPP [25], e.g., it will result in a new PPP with the half density if each node in a PPP has the probability of 0.5 to sleep independently.

Assumption 2. Wireless channel model and fixed transmit power: Wireless channel is considered as the standard pathloss multiplied by Rayleigh fading without the noise, and the transmit power of BSs is fixed.

In this paper, we consider the interference as the sum of the received signal from the interfering BSs including all the BSs other than the associated BS. As a typical cellular network is interference-limited [33], we limit the scope of this paper within interference-limited scenarios in which the interference power dominates the noise power, and thus the noise is not considered for the ease of analysis. Since the noise is ignored, the uniform transmit power adaptation does not change the SINR. Therefore, we assume that the transmit power is fixed.

Though different transmit power and different unit cost are the two main differences between macro and micro BSs captured in this paper, our model can also reflect more such as different antenna heights and antenna gains. Generally, the channel gain $g$ is modeled as $g=H d^{-\alpha} h$, where $h$ denotes the fast fading, and $H$ is predetermined by the 


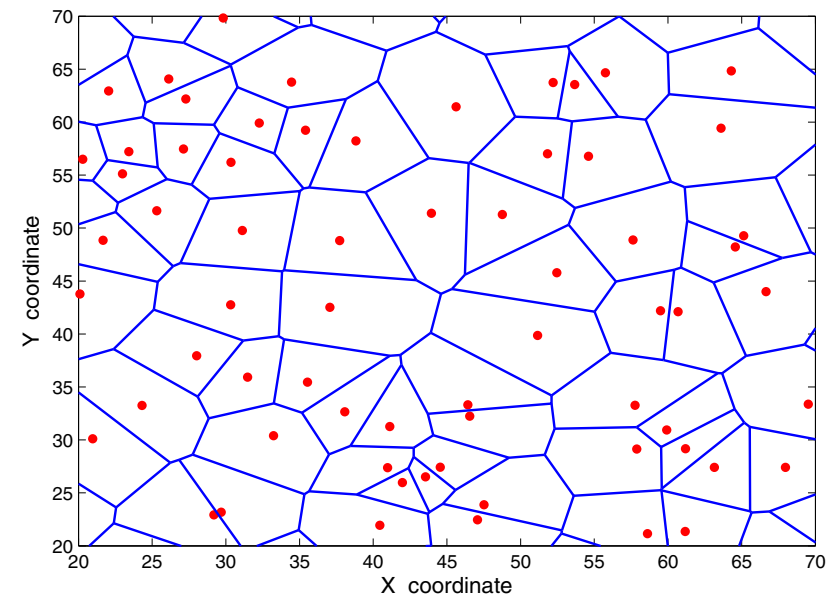

Fig. 1. Cell topology of the general homogeneous cellular network model.

antenna height and antenna gain. From a user's view, different $H$ can be reflected by different equivalent transmit power $P^{*}$, i.e., $H_{M} P_{M}=H P_{M}^{*}=H\left(\frac{H_{M}}{H} P_{M}\right)$ and $H_{m} P_{m}=$ $H P_{m}^{*}=H\left(\frac{H_{m}}{H} P_{m}\right)$, where the subscripts $M$ and $m$ denote the macro and micro BSs respectively. Moreover, in this paper we mainly consider the non-line-of-sight (NLOS) propagation, as it provides the conservative estimation for the real world. Due to the intractability, the mixed LOS/NLOS propagation is beyond the scope of this paper, which will be left for the future consideration.

Assumption 3. Frequency reuse, association and allocation schemes: Universal Frequency Reuse is adopted in both homogeneous and heterogeneous networks that each BS, either the macro or the micro, occupies the whole system spectrum. Each user is always associated to the BS with the strongest mean received signal strength, and each $B S$ allocates the resource (e.g., time slots or wireless spectrum) equally to its associated users.

The trend of cellular networks is to adopt more and more aggressive frequency reuse schemes, from the classic reuse$n$ ( $n=3,7$ typically) schemes to the current universal reuse $(n=1)$ [34] in the CDMA-based 3G networks. As the heterogenous network is an extension of the current $3 \mathrm{G}$ networks, universal frequency reuse is a promising (though not optimal) frequency planning solution for heterogeneous networks to meet the high data rate requirement. The optimal frequency reuse design is beyond the scope of this paper, and will be our future work.

For a given network, the serving area of each BS is determined by the association scheme. All the users in the serving area are associated with, and thus served by this BS. Since each user has a uniquely associated BS, the whole plane can be divived into these serving areas without overlap. In this paper, we define these serving areas as cells. Figs. 12 show the cell topology of the proposed homogeneous and heterogeneous cellular networks, respectively.

Assumption 4. Energy consumption model and user QoS requirement: The energy consumption per BS is prefixed, and

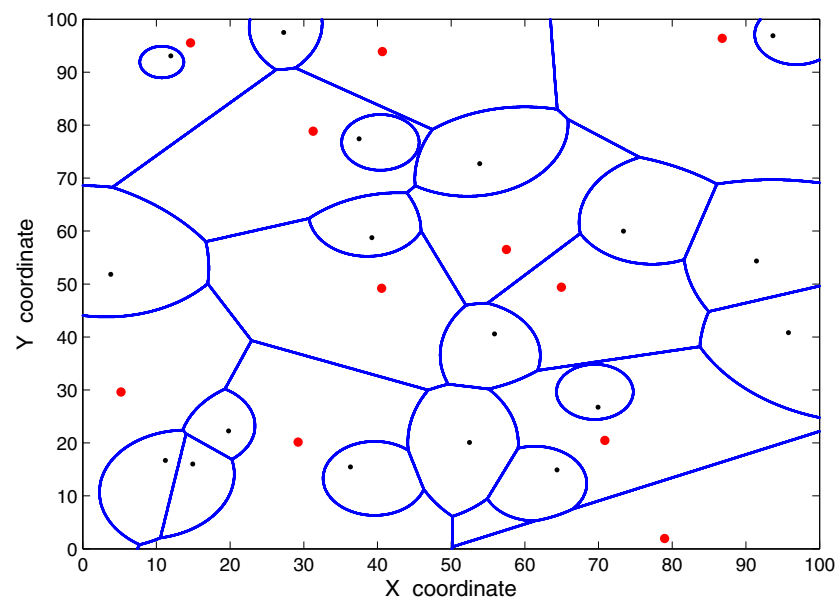

Fig. 2. Cell topology of the general heterogeneous cellular network model.

the total network energy cost is linear with the number of $B S s$ deployed or awake. The user QoS requirement is that the service outage probability in both macro and micro cells should be less than a threshold.

Generally, the energy consumption of a particular BS is a random variable, depending on many factors such as temperature, traffic load, and others. However, this random variable has a dominated statical basic part. Therefore, we assume that the unit energy cost for each BS type is fixed, which can also be viewed as the average cost since a large number of BSs are considered. For the user QoS requirement, please note that service outage occurs when the instantaneous downlink rate of a user is lower than a predefined value $u$.

With Assumptions 3 and 4, we can express the service outage constraint as

$$
G=\mathbb{E}_{\{N, A\}}\left\{\operatorname{Pr}\left(\frac{W}{N} \log _{2}(1+\operatorname{SINR})<u \mid N, A\right)\right\}<\eta,
$$

where $N(N \geq 1)$ denotes the total number of users in a certain cell, whose distribution depends on the cell size $A$. For heterogeneous scenarios, the QoS requirement is that

$$
\begin{aligned}
& G_{M}=\mathbb{E}_{\left\{N_{M}, A_{M}\right\}}\left\{\operatorname{Pr}\left(\frac{W}{N_{M}} \log _{2}(1+\mathrm{SINR})<u \mid N_{M}, A_{M}\right)\right\}<\eta, \\
& G_{m}=\mathbb{E}_{\left\{N_{m}, A_{m}\right\}}\left\{\operatorname{Pr}\left(\frac{W}{N_{m}} \log _{2}(1+\mathrm{SINR})<u \mid N_{m}, A_{m}\right)\right\}<\eta,
\end{aligned}
$$

where the index $M$ denotes macro and $m$ denotes micro. For convenience, the mathematical symbols used in this paper are summarized in Table I.

\section{Homogeneous Cellular Networks}

In homogeneous cellular networks, since the transmit power of all the BSs is the same, users are always associated with their nearest BS. For a specific BS $y$, the associated users are located within a polygonal cell $V(y)=\left\{x \in \boldsymbol{R}^{2}: n(x)=y\right\}$, where $n(x)$ denotes the nearest site function at the position $x$. These polygonal cells form the well-known PV (PoissonVoronoi) tessellation. PV tessellation is the final stage of the growth process in which seeds generated according to the 
TABLE I

SUMMARY OF NOTATION

\begin{tabular}{||c|c||}
\hline$\rho$ & BS density \\
\hline$\rho_{M}$ & macro BS density \\
\hline$\rho_{m}$ & micro BS density \\
\hline$C_{M}$ & energy cost per macro BS \\
\hline$C_{m}$ & energy cost per micro BS \\
\hline$P_{M}$ & transmit power of macro BS \\
\hline$P_{m}$ & transmit power of micro BS \\
\hline$\lambda$ & user density \\
\hline$u$ & downlink rate threshold \\
\hline$\eta$ & service outage probability threshold \\
\hline$W$ & system bandwidth \\
\hline$\alpha$ & path loss exponent, valued in $(2,4]$ \\
\hline$c$ & $c \triangleq\left(\frac{P_{M}}{P_{m}}\right)^{\frac{2}{\alpha}}$ \\
\hline$\tau$ & $\tau \triangleq \frac{\rho_{m}}{\rho_{M}}$ \\
\hline$e$ & $e \triangleq \frac{C_{m}}{C_{M}}$ \\
\hline$K$ & constant value 3.575 \\
\hline
\end{tabular}

Poisson point distribution grow simultaneously at the same isotropic rate until they grow into contact.

\section{A. Problem Formulation}

The cell size probability density function (PDF) of a PV tessellation is known to be accurately predicted by a gamma distribution [29][30] with $K=3.575$ :

$$
f(A)=\rho^{K} \frac{K^{K}}{\Gamma(K)} A^{K-1} \exp (-K \rho A),
$$

where $A$ denotes the cell size, $\rho$ is the BS density, and $\Gamma(K)=$ $\int_{0}^{\infty} x^{K-1} \exp (-x) d x$ is the gamma function.

Since the users are located according to a PPP, the number of users in a cell with cell size $A$ follows the Poisson distribution:

$$
P_{A}(n)=\frac{(\lambda A)^{n}}{n !} \exp (-\lambda A) \text {. }
$$

On the other hand, a striking property of PPPs is Slivnyak's theorem [25], which means that if we condition on having a user in a cell with cell size $A$, the number of the remaining users still follows the Poisson distribution as Eq. (4).

With the above knowledge, we get the service outage probability.

$$
\begin{aligned}
& \mathbb{E}_{\{N, A\}}\left\{\operatorname{Pr}\left(\frac{W}{N} \log _{2}(1+\mathrm{SINR})<u \mid N, A\right)\right\} \\
= & \int_{0}^{\infty} \sum_{n=0}^{\infty} \operatorname{Pr}\left(\operatorname{SINR}<2^{(n+1) \frac{u}{W}}-1 \mid n, A\right) P_{A}(n) f(A) d A \\
\approx & \int_{0}^{\infty} \sum_{n=0}^{\infty} \operatorname{Pr}\left(\operatorname{SINR}<2^{(n+1) \frac{u}{W}}-1\right) P_{A}(n) f(A) d A .
\end{aligned}
$$

The approximation is adopted similarly with [35], because it is difficult to get the SINR distribution for given cell size, as the cell size itself is difficult to calculate theoretically for given BS locations. As proved in Ref. [26], in the Rayleigh fading, no noise and PPP BS deployment scenario, the coverage probability that the SINR of a random user is greater than a threshold $T$ is expressed as

$$
\operatorname{Pr}(\operatorname{SINR} \geq T)=\frac{1}{1+T^{2 / \alpha} \int_{T^{-2 / \alpha}}^{\infty} \frac{1}{1+x^{\alpha / 2}} d x},
$$

which is independent from the BS density $\rho$ since the noise is ignored. For a special case $\alpha=4$, it can be simplified as:

$$
\operatorname{Pr}(\operatorname{SINR} \geq T)=\frac{1}{1+\sqrt{T}(\pi / 2-\arctan (1 / \sqrt{T}))} .
$$

By adopting (6) into (5) with $T=2^{(n+1) \frac{u}{W}}-1$, the service outage probability can be calculated.

To summarize, the optimal BS density problem for homogeneous cellular networks is formulated as the following:

$$
\begin{array}{ll}
\min & \rho \\
\text { s.t. } & \int_{0}^{\infty} \sum_{n=0}^{\infty} \frac{1}{1+\left(2^{(n+1) \frac{u}{W}}-1\right)^{\frac{2}{\alpha}} \int_{\left(2^{(n+1)} \frac{u}{W}-1\right)^{-\frac{2}{\alpha}} \frac{1}{1+x^{\alpha / 2}} d x}} \\
& \frac{(\lambda A)^{n}}{n !} \exp (-\lambda A) \rho^{K} \frac{K^{K}}{\Gamma(K)} A^{K-1} \exp (-K \rho A) d A \\
& \geq 1-\eta .
\end{array}
$$

\section{B. Optimal Network Density Analysis}

Problem (8) has a unique solution, since the left side of the constraint is a strictly monotone increasing function (the proof is shown in Appendix A). Thus, the optimal result can be achieved numerically through the binary search algorithm. However, the calculation of service outage probability $G$ is of high computational complexity, which consists of two embedded improper integrals and one summation of a infinite series. To exploit the impact of system parameters on the optimal result, we have derived both upper and lower bounds of the optimal BS density. Further, the upper bound is relaxed to have a closed form.

Theorem 1. The optimal BS density in the interference-limited homogeneous cellular network (8) is linear with the user density, i.e., $\rho^{*} \sim \lambda$.

Proof: Assume $\rho_{1}^{*}$ is the optimal result for user density $\lambda_{1}$, then for any other user density $\lambda_{2}, \rho_{2}=\frac{\lambda_{2}}{\lambda_{1}} \rho_{1}^{*}$ makes the QoS constraint still hold equal, which can be validated through variable substitution. Therefore, the optimal BS density for $\lambda_{2}$ is $\rho_{2}^{*}=\frac{\lambda_{2}}{\lambda_{1}} \rho_{1}^{*}$, and the optimal BS density is linear with user density.

This theorem is consistent with [23], where the authors considered the minimal inter-BS distance problem in CDMA cellular networks based on the hexagonal grid architecture, and concluded that $d^{*} \sim \lambda^{-1 / 2}$. This theorem is determined by the interference-limited nature of the cellular network, no matter what network topology it is.

1) Upper Bound:

Theorem 2. The optimal BS density in the interference-limited homogeneous cellular network (8) has an upper bound $\hat{\rho}$, which satisfies the following expression:

$$
\frac{\alpha-2}{2} 2^{\frac{-u}{w}} \sum_{m=0}^{\infty}\left(\frac{4-\alpha}{2} 2^{\frac{-u}{W}}\right)^{m}\left\{\frac{K \hat{\rho}}{\left(1-2^{(m+1) \frac{-u}{W}}\right) \lambda+K \hat{\rho}}\right\}^{K}
$$

For the special case $\alpha=4$, the upper bound has a closed-form expression:

$$
\hat{\rho}=\frac{1-2^{-\frac{u}{W}}}{K\left\{\left(2^{\frac{u}{W}}(1-\eta)\right)^{-1 / K}-1\right\}} \lambda .
$$


Further, the upper bound $\hat{\rho}$ has the following property as

$$
\lim _{\substack{u \\ W}} \frac{\hat{\rho}}{\rho^{*}}=1 .
$$

Proof: The proof is given in Appendix B.

With Theorem 2, we can further get the following less-tight but closed-form upper bound for general path loss exponent cases.

Corollary 1. The optimal BS density in the interferencelimited homogeneous cellular network (8) has a closed-form upper bound $\bar{\rho}$ which is defined as

$$
\bar{\rho} \triangleq \frac{\lambda}{\frac{W}{u} \log _{2} \frac{\frac{\alpha-2}{2}+\frac{4-\alpha}{2}(1-\eta)}{1-\eta}-1},
$$

and similarly the upper bound $\bar{\rho}$ is tight when the ratio $\frac{u}{W}$ is relatively low, i.e.,

$$
\lim _{\frac{u}{W} \rightarrow 0} \frac{\bar{\rho}}{\rho^{*}}=1 .
$$

Proof: The proof is given in Appendix C.

Corollary 1 shows that when the ratio $\frac{u}{W}$ is relatively low, the optimal BS density $\rho^{*}$ decreases with $\eta$ in the law of $\left(\log _{2} \frac{\frac{\alpha-2}{2}+\frac{4-\alpha}{2}(1-\eta)}{1-\eta}\right)^{-1}$, which reveals that the path loss exponent $\alpha$ has a great impact on the optimal result. For example, $\log _{2} \frac{\frac{\alpha-2}{2}+\frac{4-\alpha}{2}(1-\eta)}{1-\eta}$ equals to 0 when $\alpha=2$. That is because when $\alpha=2$, the sum of the interference power is infinite, and the coverage probability is always 0 as shown in (6). On the other hand, $\log _{2} \frac{\frac{\alpha-2}{2}+\frac{4-\alpha}{2}(1-\eta)}{1-\eta}$ equals to $\log _{2} \frac{1}{1-\eta}$ when $\alpha$ is 4 .

\section{2) Lower Bound:}

Theorem 3. The optimal BS density in the interference-limited homogeneous cellular network (8) has a lower bound $\check{\rho}$, which satisfies the following expression:

$$
\frac{1}{2} \sum_{m=0}^{\infty} 2^{-\left(\frac{3 u}{4 W}+1\right) m-\frac{u}{4 W}}\left\{\frac{K \check{\rho}}{\left(1-2^{-\frac{3 m+1}{4} \frac{u}{W}}\right) \lambda+K \check{\rho}}\right\}^{K}=1-\eta .
$$

Proof: The proof is given in Appendix D.

\section{Numerical Results}

1) Evaluation of upper and lower bounds: Now that we have developed the upper and lower bounds of the optimal homogeneous cellular BS density, it is important to see how tight the upper and lower bounds are. In the following, the upper and lower bounds are calculated through (9) and (14), and the optimal density is found through the binary search algorithm. According to the linearity in Theorem 1, we set the user density $\lambda=1$ as the reference value.

Fig. 3 shows how the optimal network density $\rho^{*}$ varies with the service outage threshold $\eta$. In this figure, user rate threshold $u=1 \mathrm{Mbps}$, network bandwidth $W=20 \mathrm{MHz}$ $\left(\frac{u}{W}=0.05\right)$ and the path loss exponent $\alpha=4$. We can see that both the upper bound and lower bound are close to the optimal. Furthermore, since $K\left\{\left(2^{\frac{u}{w}}(1-\eta)\right)^{-1 / K}-\right.$ $1\} \approx-\log \left(2^{\frac{u}{W}}(1-\eta)\right)$, we have that the upper bound $\hat{\rho} \sim-\frac{1-2^{-\frac{u}{W}}}{\log _{2}(1-\eta)+\frac{u}{W}}$. Due to the same trend as shown in this

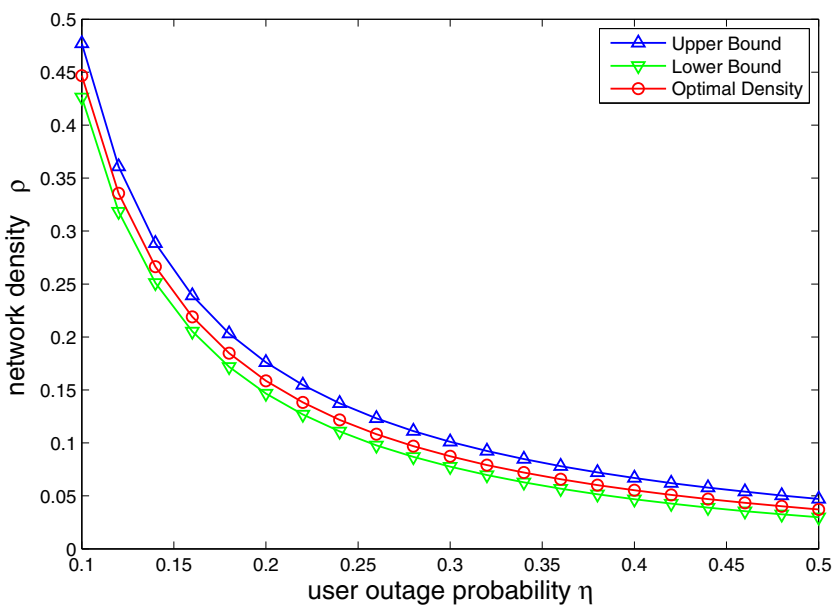

Fig. 3. Both the upper bound and the lower bound are close to the optimal density, with the parameter setting $\alpha=4, u=1 \mathrm{Mbps}$, and $W=20 \mathrm{MHz}$

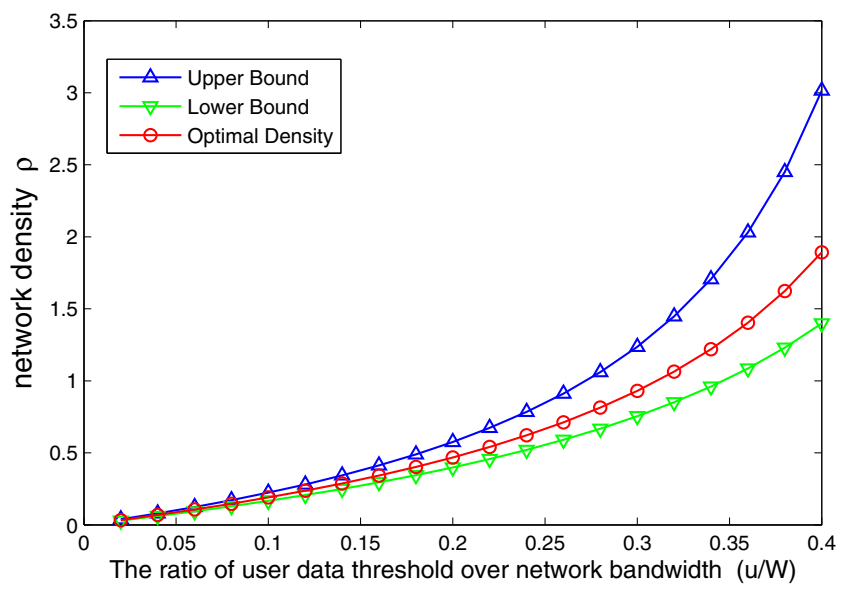

Fig. 4. The gaps of upper and lower bounds to the optimal are increasing as the ratio $\frac{u}{W}$ increases, because these densities will go to the infinity as $\frac{u}{W}$ increases, and for a sufficiently large $\frac{u}{W}$ (e.g. $\frac{u}{W} \geq 1$ ), even the infinite BS density can not satisfy the user QoS requirement. The parameter setting is $\alpha=4$ and $\eta=0.3$.

figure, it is reasonable to conjecture that $\rho^{*} \sim-\frac{1-2^{-\frac{u}{W}}}{\log _{2}(1-\eta)+\frac{u}{W}}$, and $\rho^{*} \sim-\frac{1}{\log (1-\eta)}$ when $\frac{u}{W}$ is approaching 0 .

Fig. 4 shows the impact of the ratio $\frac{u}{W}$ on the optimal density with the parameter setting $\alpha=4$ and $\eta=0.3$. We can see that the gap between the upper (lower) bound and the optimal increases as $\frac{u}{W}$ increases. The reason for this is that when $u$ is sufficiently large, even the infinite BS density cannot satisfy the QoS requirement of users with finite density. For example, the upper bound (10) is finite only when $u<$ $W \log _{2} \frac{1}{1-\eta}$. To avoid this problem, more wireless bandwidth is required, or multi-BS cooperation technology (e.g., the BS with no user inside stops radiating the interference) is adopted. However, for most of current applications, the value of $\frac{u}{W}$ is small, and the gap between the upper and lower bounds are acceptable. Remark that the parameter $u$ is not the average rate, but the minimal rate requirement.

2) Performance evaluation and discussions: In order to verify our model is applicable to realistic scenarios, following 
the documents [36][37] of EARTH project, we consider homogeneous cellular networks with voice traffic. Three scenarios are listed in [36]: dense urban, suburban and rural. In the dense urban, the user density is 11000 users $/ \mathrm{Km}^{2}$, each user brings 3 session attempts per day, and each session lasts 100 seconds on average. Therefore the equivalent active user density $\lambda_{1}=\frac{11000 \times 3 \times 100}{24 \times 60 \times 60}=38.2$ users $/ \mathrm{Km}^{2}$. Similarly, $\lambda_{2}=\frac{2000 \times 10 \times 120}{24 \times 60 \times 60}=27.8$ users $/ \mathrm{Km}^{2}$ for the suburban, and $\lambda_{3}=\frac{200 \times 6 \times 120}{24 \times 60 \times 60}=1.67 \mathrm{users} / \mathrm{Km}^{2}$ for the rural.

The rate requirement $u$ is set to $20 \mathrm{kbps}$ (voice coding rate for GSM is $13.4 \mathrm{kbps}$ ), and the downlink bandwidth $W$ is $10 \mathrm{MHz}$. According to the definition that "the system capacity is defined as the number of users in the cell when more than $95 \%$ of the users are satisfied" [36], we set the service outage threshold $\eta$ to 0.05 . The large-scale distance-dependent path loss is expressed as $L=140.7+36.7 \log _{10}(d)$, where $d$ is the distance measured in $\mathrm{Km}$, and the path loss exponent $\alpha$ is 3.67. The transmit power of BSs is $20 \mathrm{~W}$, and the terminal noise density is $-174 \mathrm{dBm} / \mathrm{Hz}$.

With the above parameter settings, the evaluation results are shown in Table II. This table shows that our noiseless assumption is acceptable for suburban and dense urban scenarios, because ignoring the noise brings little impact on the optimal result. However in the rural scenario, the noise cannot be ignored, as the required network is not dense enough to guarantee that the interference dominates the noise.

Further, the transmit power adaption is considered that when the network goes dense, the transmit power can be reduced to save energy cost. In [37], an energy consumption model for macro BSs is proposed as $C_{M}=780+28.2 P_{M}$ (in Watt). For a fixed transmit power $P_{M}$, the optimal BS density $\rho^{*}$ can be obtained numerically. Therefore, we exhaustively search for the optimal transmit power to get the minimal energy cost $C_{M} * \rho^{*}$. The numerical results are listed in Table III. Since reducing transmit power can greatly reduce the total energy consumption in this EARTH model, it is beneficial to have denser network with lower transmit power.

Table III also shows that the impact is limited, as the optimal BS density of full transmit power is close to that of transmit power adaption. The reason includes two aspects: 1) For noiselimited networks such as in rural areas, the transmit power should be large enough to guarantee coverage. Therefore, the optimal transmit power is close to full transmit power, and their optimal BS densities are also close. 2) For interferencelimited networks such as in suburban and dense urban areas, the uniform transmit power adaption has little impact on the SINR distribution, and the cellular networks are bandwidthlimited. The optimal BS density is mainly determined by user rate requirement and user density. Therefore even though the optimal transmit power is quite different from full transmit power, their optimal BS densities are also similar. Since the impact of the transmit power adaption is limited in both noiselimited and interference-limited scenarios, our assumption on fixed transmit power is meaningful on determining the optimal BS density.

\section{Heterogeneous Cellular Networks}

In this section, we turn our attention to two-tier heterogeneous cellular networks which consists of macro and micro
BSs with different cost and different coverage ability. The heterogeneous cellular network is much more complicated than the homogeneous cellular network, not just by increasing one dimension of freedom. Taking the network topology as an example, the cell size distribution in homogeneous networks depends on only one parameter: the BS density. But in heterogeneous networks, the cell size distribution depends on more, at least including the macro/micro BS density and their respective transmit power.

\section{A. User Coverage Probability}

In the homogeneous cellular networks, the user coverage probability is shown as the expression (6), which still holds in the heterogeneous cellular networks.

Lemma 1. The user SINR distribution in the heterogeneous cellular network model is the same as that in the homogeneous cellular network, i.e., the user coverage probability is still expressed as:

$$
\operatorname{Pr}(\operatorname{SINR} \geq T)=\frac{1}{1+T^{2 / \alpha} \int_{T^{-2 / \alpha}}^{\infty} \frac{1}{1+x^{\alpha / 2}} d x} .
$$

Proof: For any given user, build a coordinate system around this user, i.e., the position of this user is $(0,0)$. The position of a macro BS $i$ (the macro BS set $\Omega_{M}$ ) is $\left(x_{M i}, y_{M i}\right)$ with the distance $d_{M i}=\sqrt{x_{M i}^{2}+y_{M i}^{2}}(i=$ $0,1, \ldots)$. Similarly, $\left(x_{m i}, y_{m i}\right)$ for a micro BS $i$ (the micro BS set $\Omega_{m}$ ) with the distance $d_{m i}=\sqrt{x_{m i}^{2}+y_{m i}^{2}}$ $(i=0,1, \ldots)$. The index 0 denotes the nearest BS within its class, i.e., $d_{M 0}=\min \left\{d_{M i}\right\}$ and $d_{m 0}=\min \left\{d_{m i}\right\}$. If this user is within a macro cell, then $d_{M 0}^{-\alpha} P_{M}>d_{m 0}^{-\alpha} P_{m}$, i.e., $d_{M 0}<\left(\frac{P_{M}}{P_{m}}\right)^{1 / \alpha} d_{m 0}$. If this user is within a micro cell, then $d_{m 0}<\left(\frac{P_{m}}{P_{M}}\right)^{1 / \alpha} d_{M 0}$. Just on received signal strength, for this user, a micro BS $\left(x_{m i}, y_{m i}\right)$ is equivalent to a macro BS at the position $\left(\left(\frac{P_{M}}{P_{m}}\right)^{1 / \alpha} x_{m i},\left(\frac{P_{M}}{P_{m}}\right)^{1 / \alpha} y_{m i}\right)$. The equivalent macro BS set $\left(\frac{P_{M}}{P_{m}}\right)^{1 / \alpha} \Omega_{m}$ is still a homogeneous PPP with the density $\left(\frac{P_{m}}{P_{M}}\right)^{2 / \alpha} \rho_{m}$. As $\left(\frac{P_{M}}{P_{m}}\right)^{1 / \alpha} \Omega_{m}$ is also independent with $\Omega_{M}$, the superposition $\left(\frac{P_{M}}{P_{m}}\right)^{1 / \alpha} \Omega_{m}+\Omega_{M}$ is still a homogeneous PPP. Since this given user is associated with the nearest node in $\left(\frac{P_{M}}{P_{m}}\right)^{1 / \alpha} \Omega_{m}+\Omega_{M}$, and the SINR distribution is independent with the node density, then the SINR distribution in heterogeneous networks is the same with that in homogeneous networks.

Though the SINR distributions are the same, there is a great difference on the available bandwidth per user. Lemma 1 is consistent with [27]. With the only difference on association schemes, Ref. [27] also shows that for the uniform SINR threshold $T$, the coverage probability keeps the same, not affected by the number of tiers or their relative density and transmit power.

\section{B. Macro and Micro Cell Size Distributions}

The heterogeneous cellular network is not a PV tessellation any more, because a user may choose a macro BS far away as his home BS, not the nearest micro BS, due to their different transmit power. Actually it is a weighted PV tessellation, an extension of PV. 
TABLE II

Evaluation results on optimal BS density for these 3 scenarios (in BSs $/ \mathrm{Km}^{2}$ )

\begin{tabular}{|c|c|c|c|c|}
\hline Scenarios & $\begin{array}{c}\text { optimal BS density } \\
\text { with noise }\end{array}$ & $\begin{array}{c}\text { optimal BS density } \\
\text { without noise }\end{array}$ & $\begin{array}{c}\text { upper bound in } \\
\text { Theorem 2 }\end{array}$ & $\begin{array}{c}\text { upper bound in } \\
\text { Corollary } 1\end{array}$ \\
\hline Rural & 0.1384 & 0.0542 & 0.0551 & 0.0556 \\
Suburban & 0.9424 & 0.9017 & 0.9177 & 0.9259 \\
Dense urban & 1.2713 & 1.2390 & 1.2610 & 1.2723 \\
\hline
\end{tabular}

TABLE III

Numerical results on optimal BS density with transmit power adaption

\begin{tabular}{|c|c|c|c|}
\hline Strategy & Rural & Suburban & Dense urban \\
\hline Fixed full & $\rho^{*}=0.1384 \mathrm{BSs} / \mathrm{Km}^{2}$ & $\rho^{*}=0.9424 \mathrm{BSs} / \mathrm{Km}^{2}$ & $\rho^{*}=1.2713 \mathrm{BSs} / \mathrm{Km}^{2}$ \\
transmit power & $P_{M}=20 \mathrm{~W}$ & $P_{M}=20 \mathrm{~W}$ & $P_{M}=20 \mathrm{~W}$ \\
\hline Optimal transmit & $\rho^{*}=0.1604 \mathrm{BSs} / \mathrm{Km}^{2}$ & $\rho^{*}=1.0699 \mathrm{BSs} / \mathrm{Km}^{2}$ & $\rho^{*}=1.4121 \mathrm{BSs} / \mathrm{Km}^{2}$ \\
power adaption & $P_{M}^{*}=12.2 \mathrm{~W}$ & $P_{M}^{*}=3.8 \mathrm{~W}$ & $P_{M}^{*}=3.1 \mathrm{~W}$ \\
\hline
\end{tabular}

A weighted-PV is the final stage of the growth process in which seeds are generated according to a PPP, and each seed grows simultaneously at its own isotropic rate until they grow into contact. In mathematics, node $y_{i}$ has a growing rate (weighted factor) $w_{i}$, and its covered area is determined by

$$
V\left(y_{i}\right)=\left\{x \in \boldsymbol{R}^{2}: \frac{\left\|x-y_{i}\right\|}{w_{i}} \leq \frac{\left\|x-y_{j}\right\|}{w_{j}}, \forall j\right\} .
$$

For the homogenous scenario, each BS has the same weighted factor. For the heterogenous scenario, the weighted factor of the $i$ th $\mathrm{BS}$ is

$$
w_{i}= \begin{cases}P_{M}^{1 / \alpha}, & \text { if the } i \text { th BS is macro; } \\ P_{m}^{1 / \alpha}, & \text { otherwise. }\end{cases}
$$

However, to our best knowledge, there is no formula about the domain size distribution of the general weighted PV tessellation. In Ref. [32], the authors considered the special weighted PV tessellation in which $w_{i}$ follows a Gaussian parent distribution function independently, and concluded that the cell size distribution also follows the gamma distribution through numbers of simulations. This result and the cell size distribution of PV motivate us to do numerical fitting of the macro and micro cell size distributions with the gamma function, because PV is the extreme case of the weighted-PV as $\rho_{M}$ or $\rho_{m}$ is zero, or $P_{M}$ equals to $P_{m}$.

Lemma 2. In this heterogeneous cellular network model, the mean macro and micro cell size is expressed as

$$
\overline{A_{M}}=\mathbb{E}\left\{A_{M}\right\}=\frac{c}{c \rho_{M}+\rho_{m}}, \overline{A_{m}}=\mathbb{E}\left\{A_{m}\right\}=\frac{1}{c \rho_{M}+\rho_{m}},
$$

where, $c$ is a constant determined by the path loss exponent and their transmit power $c \triangleq\left(\frac{P_{M}}{P_{m}}\right)^{\frac{2}{\alpha}}$.

Proof: For any given user, a macro BS with the distance $d_{M}$ is equivalent to a micro BS with the distance $d_{m}=$ $\left(\frac{P_{m}}{P_{M}}\right)^{\frac{1}{\alpha}} d_{M}$ on the signal level. For this user, the heterogeneous cellular network is equivalent to the homogeneous cellular network with micro BS density $\left(\frac{P_{M}}{P_{m}}\right)^{\frac{2}{\alpha}} \rho_{M}+\rho_{m}$, i.e., $c \rho_{M}+\rho_{m}$. Therefore, a fraction $\frac{c \rho_{M}}{c \rho_{M}+\rho_{m}}$ of users take the macro BS as the home BS, and a fraction $\frac{\rho_{m}}{c \rho_{M}+\rho_{m}}$ of users take the micro BS. For a sufficiently large area $\mathcal{S}$ with acreage $S$, on average, we have,

$$
\rho_{M} S \overline{A_{M}}=\frac{c \rho_{M}}{c \rho_{M}+\rho_{m}} S, \rho_{m} S \overline{A_{m}}=\frac{\rho_{m}}{c \rho_{M}+\rho_{m}} S .
$$

Therefore, this lemma is proven.

1) Gamma Distribution Analysis: The well-known gamma distribution function is expressed as $f(x)=x^{k-1} \frac{\exp (-x / \theta)}{\theta^{k} \Gamma(k)}$ with the mean $\mathbb{E}\{x\}=k \theta$ and variance $\operatorname{Var}\{x\}=k \theta^{2}$. The parameters $(k, \theta)$ are denoted by $\left(K_{M}, \theta_{M}\right)$ for the macro cell size distribution, and $\left(K_{m}, \theta_{m}\right)$ for the micro. According to Lemma (2), we have, $K_{M} \theta_{M}=\frac{c}{c \rho_{M}+\rho_{m}}$ and $K_{m} \theta_{m}=$ $\frac{1}{c \rho_{M}+\rho_{m}}$.

Due to similarity, in the following we only focus on $\left(K_{M}, \theta_{M}\right)$. The parameters $K_{M}$ and $\theta_{M}$ are the functions of the macro BS density, the micro BS density and their transmit power. Specifically, $K_{M}\left(\rho_{M}, \rho_{m}, c\right)$ and $\theta_{M}\left(\rho_{M}, \rho_{m}, c\right)$. Define $\tau$ to be the ratio of the micro BS density over the macro BS density $\left(\tau \triangleq \frac{\rho_{m}}{\rho_{M}}\right)$. Then $K_{M}$ and $\theta_{M}$ can be reorganized as $K_{M}\left(\rho_{M}, \tau, c\right)$, and $\theta_{M}\left(\rho_{M}, \tau, c\right)$.

According to the zooming effect, i.e., $K_{M}\left(\rho_{M}, \tau, c\right) \theta_{M}\left(\rho_{M}, \tau, c\right)=\frac{1}{\rho_{M}} K_{M}(1, \tau, c) \theta_{M}(1, \tau, c)$ and $K_{M}\left(\rho_{M}, \tau, c\right) \theta_{M}^{2}\left(\rho_{M}, \tau, c\right) \stackrel{1}{=} \frac{1}{\rho_{M}^{2}} K_{M}(1, \tau, c) \theta_{M}^{2}(1, \tau, c)$, we have that $K_{M}$ only depends on $\tau$ and $c$ as $K_{M}(\tau, c)$, and $\theta_{M}\left(\rho_{M}, \tau, c\right)=\frac{1}{\rho_{M}} \theta_{M}(1, \tau, c)$. Besides, according to Lemma (2), by letting $\rho_{M}=\frac{c}{c+\tau}$, we have $K_{M}(\tau, c) \theta_{M}\left(\frac{c}{c+\tau}, \tau, c\right)=1$. Therefore, $\theta_{M}\left(\rho_{M}, \tau, c\right)=\frac{1}{\rho_{M}} \frac{c}{c+\tau} \frac{1}{K_{M}(\tau, c)}$.

Then, the gamma functions for the macro and micro cell size distribution are expressed as

$$
\begin{aligned}
& f_{M}(x)=x^{K_{M}-1} \frac{\exp \left(-\frac{c \rho_{M}+\rho_{m}}{c} K_{M} x\right)}{\left(\frac{c}{c \rho_{M}+\rho_{m}} \frac{1}{K_{M}}\right)^{K_{M}} \Gamma\left(K_{M}\right)}, \\
& f_{m}(x)=x^{K_{m}-1} \frac{\exp \left(-\left(c \rho_{M}+\rho_{m}\right) K_{m} x\right)}{\left(\frac{1}{c \rho_{M}+\rho_{m}} \frac{1}{K_{m}}\right)^{K_{m}} \Gamma\left(K_{m}\right)}
\end{aligned}
$$

where $K_{M}$ and $K_{m}$ are functions of $\tau$ and $c$, i.e., $K_{M}(\tau, c)$ and $K_{m}(\tau, c)$ respectively.

2) Case Study: Once the transmit power $P_{M}, P_{m}$ and the path loss exponent $\alpha$ are predefined, i.e., $c=\left(\frac{P_{M}}{P_{m}}\right)^{\frac{2}{\alpha}}$ is already known, $K_{M}(\tau, c)$ is simplified to be a function of $\tau$, denoted by $K_{M}(\tau)$.

As mentioned in [8], the typical transmit power of traditional macro BSs is $5 \sim 40 \mathrm{~W}$, while $0.25 \sim 2 \mathrm{~W}$ for outdoor pico BSs. Thus, in this section, we study a specific but meaningful case, in which $P_{m}$ is $10 \mathrm{~dB}$ lower than $P_{M}$, and the path loss exponent $\alpha$ is 4, i.e., $c=\left(\frac{P_{M}}{P_{m}}\right)^{\frac{2}{\alpha}}=10^{0.5}=3.1623$. 


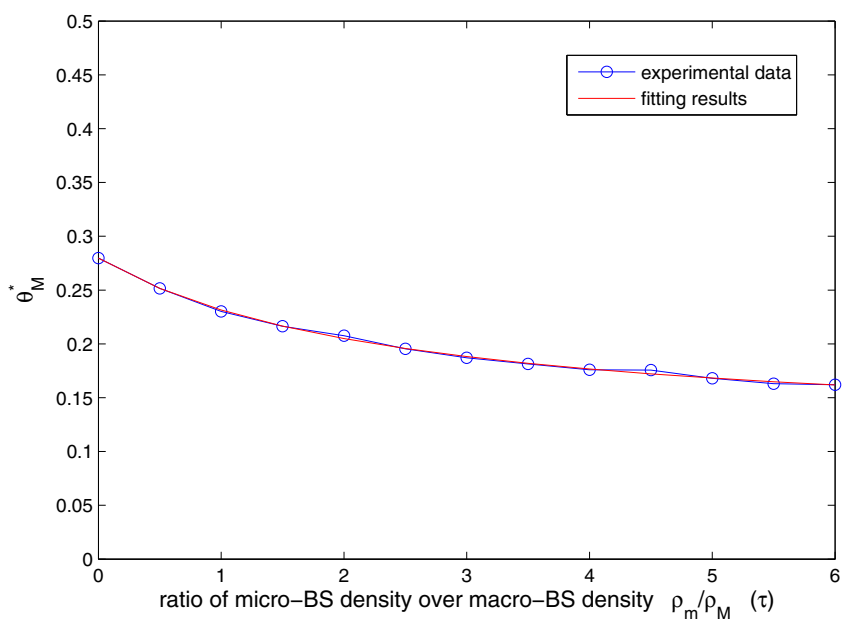

Fig. 5. Fitting chart of $\theta_{M}^{*}$. Each experimental value of $\theta_{M}^{*}$ for a given $\tau$ is obtained through 10 independent simulations, and each simulation contains more than $10^{4}$ cells with edge cells removed. The experimental results is well matched by our fitting function $\theta_{M}^{*}(\tau)=\frac{1}{3.575} \frac{1+a_{1} \tau}{1+b_{1} \tau}$ with $a_{1}=0.1673$ and $b_{1}=0.4106$ for $c=3.1623$.

Define an associated function $\theta_{M}^{*}(\tau)$ as $\theta_{M}^{*}(\tau) \triangleq \frac{1}{K_{M}(\tau)}=$ $\theta_{M}\left(\frac{c}{c+\tau}, \tau, c\right)$. Therefore, we have

$$
\theta_{M}^{*}(\tau)=\left.\operatorname{Var}\left\{A_{M}\right\}\right|_{\overline{A_{M}}=1} .
$$

The above equation (22) is adopted to get the value of $\theta_{M}$ for a given $\tau$ through simulations, as the variance of cell size is easy to get by statistics numerically.

Since PV is the extreme case of the weighted-PV when $\rho_{M}$ or $\rho_{m}$ goes to zero, the following limits should be satisfied by the fitting functions:

$$
\lim _{\tau \rightarrow 0} \theta_{M}^{*}(\tau)=\frac{1}{K}, \lim _{\tau \rightarrow \infty} \theta_{m}^{*}(\tau)=\frac{1}{K} .
$$

For this specific case, our simulation results show that $\theta_{M}^{*}$ and $\theta_{m}^{*}$ are well predicted by:

$$
\theta_{M}^{*}(\tau)=\frac{1}{3.575} \frac{1+0.1673 \tau}{1+0.4106 \tau} ; \theta_{m}^{*}(\tau)=\frac{1}{3.575} \frac{\tau+5.1952}{\tau+2.5327}
$$

Finally, for clarity, we present the complete fitting functions of the macro and micro cell size distribution for this case study as follows:

$$
f_{M}(x)=x^{K_{M}-1} \frac{\exp \left(-\frac{c \rho_{M}+\rho_{m}}{c} K_{M} x\right)}{\left(\frac{c}{c \rho_{M}+\rho_{m}} \frac{1}{K_{M}}\right)^{K_{M}} \Gamma\left(K_{M}\right)},
$$

where, $K_{M}=3.575 \frac{1+0.4106 \tau}{1+0.1673 \tau}=3.575 \frac{\rho_{M}+0.4106 \rho_{m}}{\rho_{M}+0.1673 \rho_{m}}$;

$$
f_{m}(x)=x^{K_{m}-1} \frac{\exp \left(-\left(c \rho_{M}+\rho_{m}\right) K_{m} x\right)}{\left(\frac{1}{c \rho_{M}+\rho_{m}} \frac{1}{K_{m}}\right)^{K_{m}} \Gamma\left(K_{m}\right)},
$$

where, $K_{m}=3.575 \frac{\tau+2.5327}{\tau+5.1952}=3.575 \frac{\rho_{m}+2.5327 \rho_{M}}{\rho_{m}+5.1952 \rho_{M}}$.

3) Fitting Charts and Verifications: Figs. 5 and 6 are the fitting charts of $\theta_{M}^{*}(\tau)$ and $\theta_{m}^{*}(\tau)$ respectively. In the both two figures, each experimental result is obtained through 10 independent simulations, and each simulation contains more than $10^{4}$ cells with edge cells removed. From these fitting charts, we can see that the experimental results are well matched by our fitting functions $\theta_{M}^{*}(\tau)$ and $\theta_{m}^{*}(\tau)$.

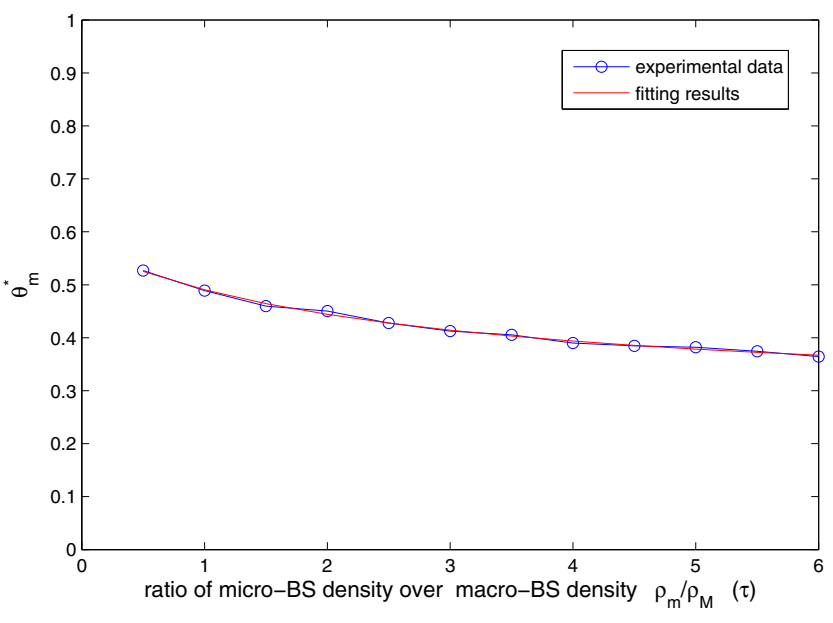

Fig. 6. Fitting chart of $\theta_{m}^{*}$. Each experimental value of $\theta_{m}^{*}$ for a given $\tau$ is obtained through 10 independent simulations, and each simulation contains more than $10^{4}$ cells with edge cells removed. The experimental results is well matched by our fitting function $\theta_{m}^{*}(\tau)=\frac{1}{3.575} \frac{\tau+a_{2}}{\tau+b_{2}}$ with $a_{2}=5.1952$ and $b_{2}=2.5327$ for $c=3.1623$.

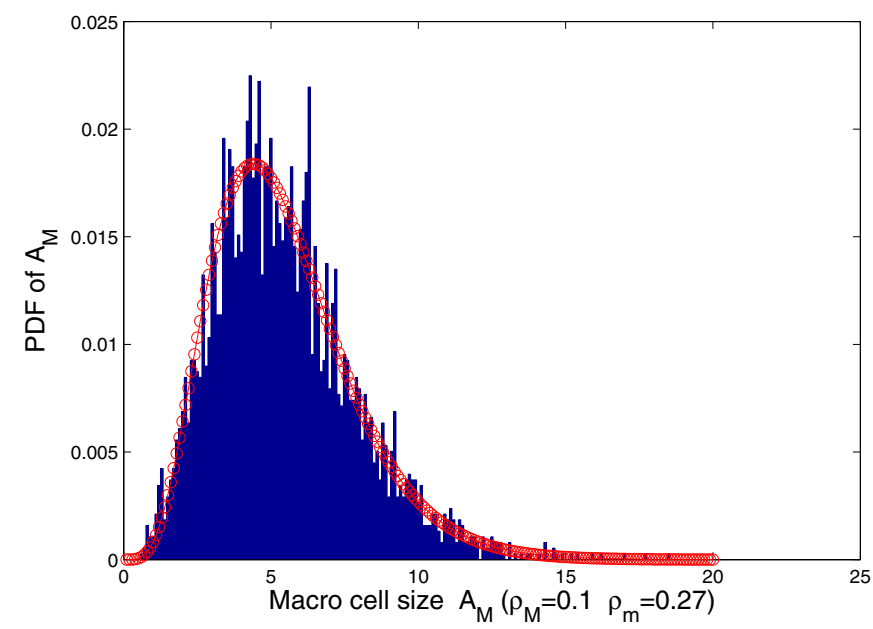

Fig. 7. Verification chart of the macro cell size distribution with parameters setting $\rho_{M}=0.1$ and $\rho_{m}=0.27$.

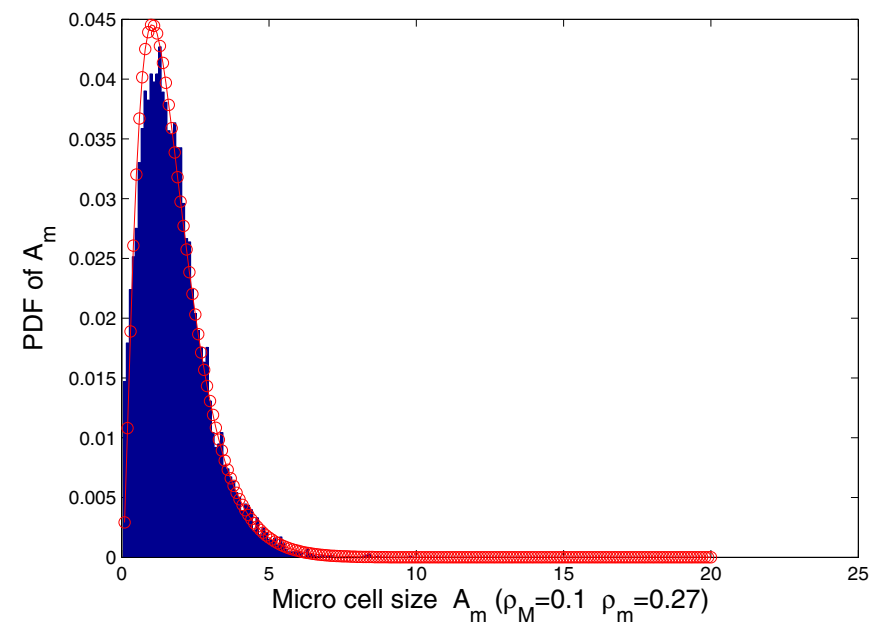

Fig. 8. Verification chart of the micro cell size distribution with parameters setting $\rho_{M}=0.1$ and $\rho_{m}=0.27$. 
Further, we still need to verify that the distributions are gamma function-like, i.e., the macro and micro cell size are accurately predicted by (25) and (26) respectively. Figs. 7 and 8 are verification charts of the macro and micro cell size distribution respectively, with the parameter setting $\tau=2.7$. The red lines are from our fitting functions. These figures show that the macro and micro cell size distributions are accurately predicted by our fitting functions.

\section{Problem Setup and Formulation}

In this paper, we focus on two important issues in heterogeneous networks: capacity extension through BS deployment, and energy saving through BS switching.

1) Capacity extension: Consider an existing cellular network which is already deployed with macro and micro BSs with densities $\rho_{0}$ and $\rho_{1}$ ( $\rho_{1}$ can be 0$)$ respectively. The coverage is guaranteed by the existing macro BSs. However, this cellular network requires to extend its capacity to satisfy the increasing QoS requirement, and a direct approach is to deploy more BSs. Then to minimize the total cost, one would concern how many macro and micro BSs should be added.

2) Energy saving: Consider an existing cellular network which consists of macro and micro BSs with densities $\rho_{2}$ and $\rho_{3}$ respectively. If the BS sleeping is a feasible option to reduce the energy consumption, one may decide how many macro and micro BSs can be switched off when the traffic load is low (e.g., at night). To avoid the coverage hole caused by BS sleeping, some macro BSs, called "coverage BSs", cannot be switched off, and their density is $\rho_{0}$.

Since BS deployment and switching are two operations that are directly related with BS density, these two problems can be generalized into a uniform BS density problem (27).

For capacity extension, the objective is to minimize $C_{M}\left(\rho_{M}-\rho_{0}\right)+C_{m}\left(\rho_{m}-\rho_{1}\right)$. For energy saving, the objective is to maximize $C_{M}\left(\rho_{2}-\rho_{M}\right)+C_{m}\left(\rho_{3}-\rho_{m}\right)$. Since the values of $\left(\rho_{0}, \rho_{1}, \rho_{2}, \rho_{3}\right)$ are prefixed, both the objectives are equivalent to minimizing $C_{M} \rho_{M}+C_{m} \rho_{m}$, or $\rho_{M}+e \rho_{m}$. Denote the optimal result of (27) by $\left(\rho_{M}^{*}, \rho_{m}^{*}\right)$. Then the deployment strategy for capacity extension is to deploy macro BSs with the density $\rho_{M}^{*}-\rho_{0}$ and micro BSs with the density $\rho_{m}^{*}-\rho_{1}$ independently, because the superposition of two independent PPPs is still a PPP. Similarly the BS sleeping for energy saving is to switch off the macro BSs other than coverage $B S s$ with the probability $\frac{\rho_{2}-\rho_{M}^{*}}{\rho_{2}-\rho_{0}}$ and the micro BSs with the probability $\frac{\rho_{3}-\rho_{m}^{*}}{\rho_{3}}$ independently, as the independent thinning of a PPP is also a PPP.

\section{Optimal Heterogeneous Network Density Analysis}

Based on the previous analysis of Corollary 1 , we can obtain the following inequations,

$$
\begin{aligned}
G_{M} & \leq 1-\frac{\frac{\alpha-2}{2} 2^{-\frac{u}{W}\left(1+\frac{c \lambda}{c \rho_{M}+\rho_{m}}\right)}}{1-\frac{4-\alpha}{2} 2^{-\frac{u}{W}\left(1+\frac{c \lambda}{c \rho_{M}+\rho_{m}}\right)}}, \\
G_{m} & \leq 1-\frac{\frac{\alpha-2}{2} 2^{-\frac{u}{W}\left(1+\frac{\lambda}{c \rho_{M}+\rho_{m}}\right)}}{1-\frac{4-\alpha}{2} 2^{-\frac{u}{W}\left(1+\frac{\lambda}{c \rho_{M}+\rho_{m}}\right)}} .
\end{aligned}
$$

With these inequations, the original problem (27) can be reinforced as

$$
\begin{array}{ll}
\min & \rho_{M}+e \rho_{m} \\
\text { s.t. } & 1-\frac{\frac{\alpha-2}{2} 2^{-\frac{u}{W}\left(1+\frac{c \lambda}{c \rho_{M}+\rho_{m}}\right)}}{1-\frac{4-\alpha}{2} 2^{-\frac{u}{W}\left(1+\frac{c \lambda}{c \rho_{M}+\rho_{m}}\right)}} \leq \eta, \\
& 1-\frac{\frac{\alpha-2}{2} 2^{-\frac{u}{W}\left(1+\frac{\lambda}{\rho_{M}+\rho_{m}}\right)}}{1-\frac{4-\alpha}{2} 2^{-\frac{u}{W}\left(1+\frac{\lambda}{c \rho_{M}+\rho_{m}}\right)}} \leq \eta, \\
& \rho_{0} \leq \rho_{M} \leq \rho_{2}, \\
& \rho_{1} \leq \rho_{m} \leq \rho_{3},
\end{array}
$$

which is equivalent to

$$
\begin{array}{ll}
\min & \rho_{M}+e \rho_{m} \\
\text { s.t. } & 1-\frac{\frac{\alpha-2}{2} 2^{-\frac{u}{W}\left(1+\frac{c \lambda}{c \rho_{M}+\rho_{m}}\right)}}{1-\frac{4-\alpha}{2} 2^{-\frac{u}{W}\left(1+\frac{c \lambda}{c \rho_{M}+\rho_{m}}\right)}} \leq \eta, \\
& \rho_{0} \leq \rho_{M} \leq \rho_{2} \\
& \rho_{1} \leq \rho_{m} \leq \rho_{3} .
\end{array}
$$

By letting the first constraint of (30) be equal, we have,

$$
\rho_{M}=\frac{c}{c+\tau} \frac{\lambda}{\frac{W}{u} \log _{2} \frac{\frac{\alpha-2}{2}+\frac{4-\alpha}{2}(1-\eta)}{1-\eta}-1},
$$

which leads to the following equivalent problem:

$$
\begin{array}{ll}
\min & \frac{1+e \tau}{1+\frac{1}{c} \tau} \frac{\lambda}{\frac{W}{u} \log _{2} \frac{\frac{\alpha-2}{2}+\frac{4-\alpha}{2}(1-\eta)}{1-\eta}-1} \\
\text { s.t. } & \rho_{0} \leq \frac{c}{c+\tau} \frac{\lambda}{\frac{W}{u} \log _{2} \frac{\frac{\alpha-2}{2}+\frac{4-\alpha}{2}(1-\eta)}{1-\eta}-1} \leq \rho_{2}, \\
& \rho_{1} \leq \frac{c \tau}{c+\tau} \frac{\lambda}{\frac{W}{u} \log _{2} \frac{\frac{\alpha-2}{2}+\frac{4-\alpha}{2}(1-\eta)}{1-\eta}-1} \leq \rho_{3} .
\end{array}
$$

The function $\rho_{M}(\tau)$ in (31) is decreasing, and $\rho_{m}(\tau)=$ $\tau \rho_{M}(\tau)$ is increasing with $\tau$. Therefore, by denoting the value of $\tau$ that makes the constraint with $\rho_{i}$ hold equal by $\tau_{i}(i=$ $0,1,2,3)$, the reinforced problem then can be simplified as

$$
\begin{array}{ll}
\min & \frac{1+e \tau}{1+\frac{1}{c} \tau} \frac{\lambda}{\frac{W}{u} \log _{2} \frac{\frac{\alpha-2}{2}+\frac{4-\alpha}{2}(1-\eta)}{1-\eta}-1} \\
\text { s.t. } & \max \left\{\tau_{1}, \tau_{2}\right\} \leq \tau \leq \min \left\{\tau_{0}, \tau_{3}\right\},
\end{array}
$$

which reveals that when $e$ is larger than $\frac{1}{c}$, the objective is an increasing function, and the optimal $\tau^{*}$ should be as small as possible; otherwise, $\tau^{*}$ should be as large as possible.

$$
\tau^{*}= \begin{cases}\min \left\{\tau_{0}, \tau_{3}\right\}, & 0 \leq e<\frac{1}{c} \\ \max \left\{\tau_{1}, \tau_{2}\right\}, & \frac{1}{c}<e .\end{cases}
$$

Specifically, for capacity extension, if $e>\frac{1}{c}$, it is better to deploy more macro BS sites, while if $e<\frac{1}{c}$, the optimal strategy is to deploy micro BS sites. For energy saving, if $e>\frac{1}{c}$, it is better to switch off certain micro BS sites, while if $e<\frac{1}{c}$, the optimal strategy is to switch off certain macro BS sites. 


$$
\begin{array}{ll}
\min & \rho_{M}+e \rho_{m} \\
\text { s.t. } & G_{M}=\mathbb{E}_{\left\{N_{M}, A_{M}\right\}}\left\{\operatorname{Pr}\left(\frac{W}{N_{M}} \log _{2}(1+\mathrm{SINR})<u \mid N_{M}, A_{M}\right)\right\}<\eta, \\
& G_{m}=\mathbb{E}_{\left\{N_{m}, A_{m}\right\}}\left\{\operatorname{Pr}\left(\frac{W}{N_{m}} \log _{2}(1+\mathrm{SINR})<u \mid N_{m}, A_{m}\right)\right\}<\eta, \\
& \rho_{0} \leq \rho_{M} \leq \rho_{2}, \\
& \rho_{1} \leq \rho_{m} \leq \rho_{3} .
\end{array}
$$

With the definition of $\tau_{i}$, we can further get

$$
\tau_{i}= \begin{cases}\frac{\lambda}{\rho_{i}} \frac{c}{\frac{W}{u} \log _{2} \frac{\frac{\alpha-2}{2}+\frac{4-\alpha}{2}(1-\eta)}{1-\eta}-1}-c, & i=0,2 ; \\ \frac{1}{\rho_{i}} \frac{\frac{1}{u} \log _{2} \frac{\frac{\alpha-2}{2}+\frac{4-\alpha}{2}(1-\eta)}{1-\eta}-1}{c}, & i=1,3 .\end{cases}
$$

Therefore, the reinforced problem (29) has a closed-form solution by jointing (31), (34) and (35). This closed-form solution also provides a conservative estimate on the optimal result of the original problem (27).

\section{E. Numerical Results and Discussion}

1) Validation of our analysis: Fig. 9 takes the capacity extension as an example to compare the achievable network costs of the reinforced problem (29) and the original problem (27), which are obtained through the exhaustive search. The parameter setting is that $u=1 \mathrm{Mbps}, W=20 \mathrm{MHz}$, $P_{m}=0.1 P_{M}$, and $\alpha=4$. Consider that there has been a homogenous cellular network with macro BSs with the density $\rho_{0}=\frac{1-2^{-\frac{u}{W}}}{K\left\{\left(2 \frac{u}{W}(1-\eta)\right)^{-1 / K}-1\right\}} \lambda_{0}=0.4770$ per unit area, where $\lambda_{0}=1$ per unit area. However, capacity extension is needed, since $\lambda=2 \lambda_{0}$. This figure verifies our rule on BS type selection (34). Specifically, when $e \leq 0.3$, the optimal BS type is the micro, therefore, the optimal network cost is linear with $e$; when $e \geq 0.35$, the optimal network cost is constant, for the optimal BS type is the macro.

2) Performance evaluation and discussions: In this subsection, we evaluate the energy consumption performance of the heterogeneous networks and BS sleeping in a realistic dense urban scenario. Similarly, voice traffic is considered but with the time-varying property. The time-varying traffic profile is configured according to [38] that the whole day is split into hours, and in each hour the traffic load keeps constant. Specifically, denote the active user density at time $t$ by $\lambda_{t}$ and the peak density by $\lambda_{p}$. Then, the normalized ratio $\frac{\lambda_{t}}{\lambda_{p}}$ is listed in Table IV [38]. Jointly with the parameters in [36], to make the total traffic volume equal, $\lambda_{p}$ in the dense urban scenario is 74.3 users $/ \mathrm{Km}^{2}$. The other configurations such as channel gain and noise power are the same with those in Subsection III-C2 for default.

The power consumption for macro BSs is $C_{M}=780+$ $28.2 P_{M}$ and that for micro BSs is $C_{m}=112+5.2 P_{m}$, in which $P_{M} \leq 20$ and $P_{m} \leq 6.3$ [37]. Since the cell size distribution of only the specific case with parameter $c=3.1623$ is well studied, in the following we fix the transmit power of BSs as $P_{M}=20$ and $P_{m}=2.42$ (in

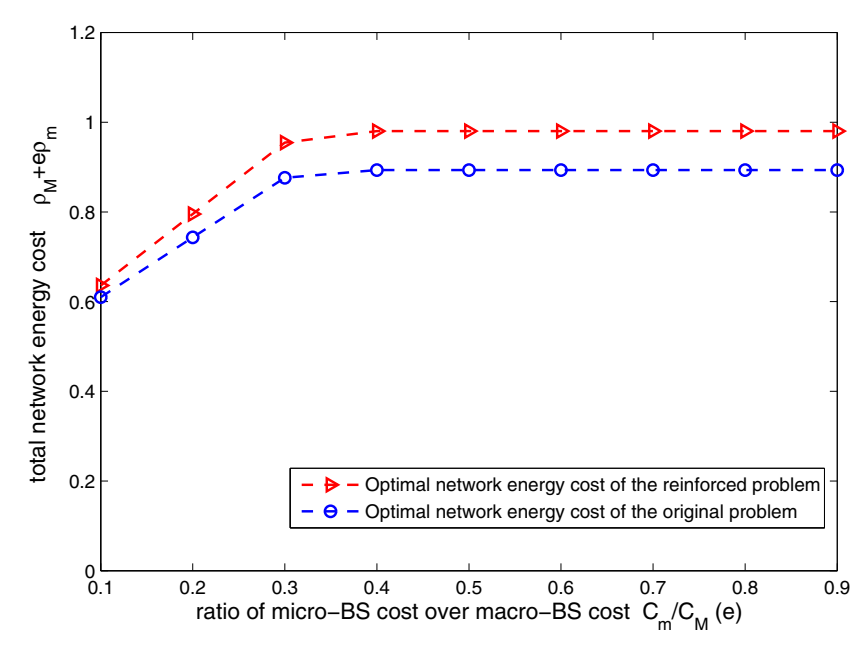

Fig. 9. The optimal network energy costs of the reinforced problem and the original problem. An example of capacity extension: $u=1 \mathrm{Mbps}, W=20 \mathrm{MHz}$, $\eta=0.1, P_{m}=0.1 P_{M}, \alpha=4, \lambda=2$ per unit area, $\rho_{0}=0.4770$ per unit area, $\rho_{1}=0$ per unit area, and $\rho_{2}=\rho_{3}=\infty$ per unit area.

Watt). In such case, $e=\frac{112+5.2 P_{m}}{780+28.2 P_{M}}=0.0927$ is less than $c^{-1}=0.3162$, and thus micro BSs are preferable, i.e., it is optimal to deploy micro BSs or to switch off macro BSs (if possible) with higher priority. For comparison, the macro-only homogeneous cellular network with no BS sleeping is defined as the reference system.

(1) Capacity extension: Consider a macro-only homogeneous cellular network that requires capacity extension to satisfy the peak traffic, and the initial density is $1.0 \mathrm{BSs} / \mathrm{Km}^{2}$. If the only macro BSs are deployed, then the optimal newlyadded macro BS density is $1.4286 \mathrm{BSs} / \mathrm{Km}^{2}$, and the final network energy cost is $3.26 \mathrm{KW} / \mathrm{Km}^{2}$. On the other hand if micro BSs can be deployed, then the optimal strategy is to add micro BSs with the density $4.5491 \mathrm{BSs} / \mathrm{Km}^{2}(4.4898$ $\mathrm{BSs} / \mathrm{Km}^{2}$ if noise is ignored), and the final network energy cost is $1.91 \mathrm{KW} / \mathrm{Km}^{2}$. Therefore compared to the reference system, deploying heterogeneous cellular networks can reduce about $41.4 \%$ of the total network consumption.

(2) Energy saving: For the newly-deployed heterogeneous network with the initial BS density combination $\left(\rho_{2}, \rho_{3}\right)=$ $(1.0,4.5491) \mathrm{BSs} / \mathrm{Km}^{2}$, consider BS sleeping to further reduce the energy consumption when traffic is off the peak. Since the network in the rural area is coverage-limited, $\rho_{0}$ is set to $0.1384 \mathrm{BSs} / \mathrm{Km}^{2}$ according to the previous results obtained in Subsection III-C2. The BS sleeping strategy is shown in Table IV, and the average network energy cost is 0.82 
$\mathrm{KW} / \mathrm{Km}^{2}$. Therefore compared to the reference system, the heterogeneous cellular network with BS sleeping can reduce about $75 \%$ of the total network consumption.

\section{CONClusion}

In this paper, we adopt stochastic geometry theory to analyze the optimal BS density for both homogeneous and heterogeneous cellular networks with service outage probability constraint.

For homogeneous cellular networks, we derive the upper and lower bounds of the optimal BS density, and numerical results demonstrate that both the upper and lower bounds are tight. Moreover, the upper bound can be relaxed to have a closed form.

For heterogeneous cellular networks, we do numerical fitting of the macro and micro cell size distribution with the Gamma distribution, and verify that the distribution is accurately predicted by Gamma functions. Then, we solve two important issues: capacity extension and energy saving, and explore a rule to determine which type of BSs should be deployed or slept with higher priority. Based on our PPP model and universal frequency reuse condition, the rule reveals that if $e$ is lower than a threshold $1 / c$, deploying or switching on more micro BSs is more beneficial. Otherwise, the optimal choice is the opposite. Based on EARTH parameters, numerical results show that compared to the traditional homogeneous cellular network, the heterogeneous cellular network with BS sleeping can reduce the total energy consumption by up to $75 \%$. Since our analysis is based on simplified assumptions, the future work will focus on the joint transmit power and BS density optimization with more realistic conditions.

\section{APPENDIX A}

Denote $\operatorname{Pr}\left(\frac{W}{n+1} \log _{2}(1+\operatorname{SINR})<u\right)$ by $R(n)$, which is a decreasing function of $n$, i.e., $R(n+1)<R(n)(n=0,1, \ldots)$. Then the service outage probability $G$ is expressed as (36). By letting $t=\rho A$, then

$$
G=\int_{0}^{\infty} \sum_{n=0}^{\infty} R(n) \frac{\left(\frac{\lambda}{\rho} t\right)^{n}}{n !} \exp \left(-\frac{\lambda}{\rho} t\right) \frac{K^{K}}{\Gamma(K)} t^{K-1} \exp (-K t) d t .
$$

Eq. (36) shows that the service outage probability only depends on the ratio $a=\frac{\lambda}{\rho}$, and we only need to prove that $y=\sum_{n=0}^{\infty} R(n) \frac{(a t)^{n}}{n !} \exp (-a t)$ is decreasing with $a$.

$$
\begin{aligned}
\frac{\partial y}{\partial a} & =\frac{\partial}{\partial a}\left[R(0) \exp (-a t)+\sum_{n=1}^{\infty} R(n) \frac{(a t)^{n}}{n !} \exp (-a t)\right] \\
& =\sum_{n=0}^{\infty}(R(n+1)-R(n)) \frac{(a t)^{n}}{n !} \exp (-a t) t \\
& <0 .
\end{aligned}
$$

Therefore the service outage probability is strictly decreasing with BS density.

\section{APPENDIX B}

Definition 1. Define ' $\lesssim$ ' as a relation between two functions $f(x, y)$ and $g(x, y)$, if the following is satisfied that

$$
f(x, y) \lesssim_{y} g(x, y) \Leftrightarrow \begin{cases}\lim _{y \rightarrow 0} \frac{f(x, y)}{g(x, y)}=1, & \forall x \in \Omega_{x} \\ f(x, y) \leq g(x, y), & \forall\{x, y\} \in \Omega_{x, y}\end{cases}
$$

where, $\Omega_{x}$ denotes the domain of $x$, and similarly for $\Omega_{x, y}$.

Define ' $\gtrsim$ ' as that $f(x, y) \gtrsim y g(x, y)$ if and only if $g(x, y) \lesssim_{y} f(x, y)$ is satisfied.

With the above definition, Theorem 2 is proved as follows.

Proof: Based on Eq. (6), let $T=2^{(n+1) \frac{u}{W}}-1$ and we have,

$$
\begin{aligned}
\operatorname{Pr}(\operatorname{SINR} \geq T) & =\frac{1}{1+T^{2 / \alpha} \int_{T^{-2 / \alpha}}^{\infty} \frac{1}{1+x^{\alpha / 2}} d x} \\
& \gtrsim \frac{u}{W} \frac{1}{1+T^{2 / \alpha} \int_{T^{-2 / \alpha}}^{\infty} x^{-\alpha / 2} d x}=\frac{1}{1+\frac{2}{\alpha-2} T} \\
& =\frac{\alpha-2}{2} 2^{-(n+1) \frac{u}{W}} \frac{1}{1-\frac{4-\alpha}{2} 2^{-(n+1) \frac{u}{W}}} \\
& \stackrel{(a)}{=} \frac{\alpha-2}{2} 2^{-\frac{u}{W}} \sum_{m=0}^{\infty}\left(\frac{4-\alpha}{2}\right)^{m} 2^{-m \frac{u}{W}} 2^{-(m+1) \frac{u}{W} n}
\end{aligned}
$$

Eq. (a) holds, because generally the path loss exponent $\alpha \in$ $(2,4]$ makes $\frac{4-\alpha}{2} \in[0,1)$.

Therefore,

$$
\begin{aligned}
& \sum_{n=0}^{\infty} \operatorname{Pr}\left(\operatorname{SINR} \geq 2^{(n+1) \frac{u}{W}}-1\right) P_{A}(n) \\
& \gtrsim_{\frac{u}{W}} \frac{\alpha-2}{2} 2^{\frac{-u}{W}} \sum_{m=0}^{\infty}\left(\frac{4-\alpha}{2} 2^{\frac{-u}{W}}\right)^{m} \exp \left(\left(2^{-(m+1) \frac{u}{W}}-1\right) \lambda A\right),
\end{aligned}
$$

and finally,

$$
\begin{aligned}
& \int_{0}^{\infty} \sum_{n=0}^{\infty} \operatorname{Pr}\left(\operatorname{SINR} \geq 2^{(n+1) \frac{u}{W}}-1\right) P_{A}(n) f(A) d A \\
& \gtrsim_{\frac{u}{W}} \frac{\alpha-2}{2} 2^{\frac{-u}{W}} \sum_{m=0}^{\infty}\left(\frac{4-\alpha}{2} 2^{\frac{-u}{W}}\right)^{m}\left\{\frac{K \rho}{\left(1-2^{-(m+1) \frac{u}{W}}\right) \lambda+K \rho}\right\}^{K} .
\end{aligned}
$$

By letting $\rho=\rho^{*}$, we have,

$$
1-\eta \gtrsim \frac{u}{W} \frac{\alpha-2}{2} 2 \frac{-u}{W} \sum_{m=0}^{\infty}\left(\frac{4-\alpha}{2} 2 \frac{-u}{W}\right)^{m}\left\{\frac{K \rho^{*}}{\left(1-2^{-(m+1)} \frac{u}{W}\right) \lambda+K \rho^{*}}\right\}_{(42)}^{K} .
$$

Denoting the value by $\hat{\rho}$ which satisfies (9) and due to the monotonicity, $\rho^{*} \lesssim \frac{u}{W} \hat{\rho}$ is proven, i.e., $\rho^{*} \leq \hat{\rho}$ and $\lim _{\frac{u}{W} \rightarrow 0} \frac{\hat{\rho}}{\rho^{*}}=$ 1.

Moreover, for the special case $\alpha=4$, (9) can be rewritten as Eq. (10). 
TABLE IV

BS SLEEPING STRATEGY IN THE DENSE URBAN SCENARIO

\begin{tabular}{ccccc}
\hline Time & $\begin{array}{c}\text { normalized traffic } \\
\text { to the peak }\end{array}$ & $\begin{array}{c}\text { macro BS sleeping } \\
\text { probability } \frac{\rho_{2}-\rho_{M}^{*}}{\rho_{2}-\rho_{0}}\end{array}$ & $\begin{array}{c}\text { micro BS sleeping } \\
\text { probability } \frac{\rho_{3}-\rho_{m}^{*}}{\rho_{3}}\end{array}$ & $\begin{array}{c}\text { energy consumption } \\
\left(\mathrm{KW} / \mathrm{Km}^{2}\right)\end{array}$ \\
\hline 09:00-11:00 & $90 \%$ & $28.1 \%$ & $0 \%$ & 1.59 \\
11:00-13:00 & $100 \%$ & $0 \%$ & $0 \%$ & 1.91 \\
$13: 00-15: 00$ & $70 \%$ & $84.2 \%$ & $0 \%$ & 0.95 \\
$15: 00-18: 00$ & $80 \%$ & $56.2 \%$ & $0 \%$ & 1.27 \\
$18: 00-23: 00$ & $55 \%$ & $100 \%$ & $15.5 \%$ & 0.68 \\
23:00-09:00 & $20 \%$ & $100 \%$ & $72.5 \%$ & 0.36 \\
\hline
\end{tabular}

$$
G=\int_{0}^{\infty} \sum_{n=0}^{\infty} R(n) \frac{(\lambda A)^{n}}{n !} \exp (-\lambda A) \rho^{K} \frac{K^{K}}{\Gamma(K)} A^{K-1} \exp (-K \rho A) d A
$$

\section{APPENDix C}

In (41), due to the well-known exponential limit,

$$
\begin{aligned}
\left\{\frac{K \rho}{\left(1-2^{-(m+1) \frac{u}{W}}\right) \lambda+K \rho}\right\}^{K} & =\left\{1+\frac{1}{\frac{1}{\left(1-2^{-(m+1)} \frac{u}{W}\right) \lambda} K \rho}\right\}^{-K} \\
& \gtrsim \frac{u}{W} \exp \left\{-\frac{\left(1-2^{-(m+1)} \frac{u}{W}\right) \lambda}{\rho}\right\} \\
& \gtrsim \frac{u}{W} \exp \left\{-(m+1) \frac{u}{W} \frac{\lambda}{\rho} \log 2\right\} \\
& =2^{-(m+1) \frac{u}{W} \frac{\lambda}{\rho} .}
\end{aligned}
$$

Therefore by adopting (43) into (41),

$$
\begin{aligned}
& \int_{0}^{\infty} \sum_{n=0}^{\infty} \operatorname{Pr}\left(\operatorname{SINR} \geq 2^{(n+1) \frac{u}{W}}-1\right) P_{A}(n) f(A) d A \\
& \gtrsim_{W} \frac{\alpha-2}{2} 2^{-\frac{u}{W}\left(1+\frac{\lambda}{\rho}\right)} \sum_{m=0}^{\infty}\left(\frac{4-\alpha}{2} 2^{-\frac{u}{W}\left(1+\frac{\lambda}{\rho}\right)}\right)^{m} \\
& =\frac{\frac{\alpha-2}{2} 2^{-\frac{u}{W}\left(1+\frac{\lambda}{\rho}\right)}}{1-\frac{4-\alpha}{2} 2^{-\frac{u}{W}\left(1+\frac{\lambda}{\rho}\right)}} .
\end{aligned}
$$

By letting $\rho=\rho^{*}$, we have $\rho^{*} \lesssim \frac{u}{W} \bar{\rho}$, and Corollary 1 is proven.

\section{APPENDIX D}

Before proving Theorem 3, we first introduce the following lemma.

Lemma 3. The coverage probability in (6) is increasing with the path loss exponent $\alpha$.

Proof: Assume the path loss exponent $\alpha_{1}<\alpha_{2}$. Once all the BSs have been already located, for any given user, the coverage probability is

$\operatorname{Pr}(\operatorname{SINR} \geq T)=\operatorname{Pr}\left\{\left(h_{0}, h_{1}, \ldots, h_{\infty}\right): h_{0} d_{0}^{-\alpha} / \sum_{i=1}^{\infty} h_{i} d_{i}^{-\alpha} \geq T\right\}$

where $h_{i}$ is the Rayleigh fading factor (0 represents the nearest BS). Since $d_{0} \leq d_{i}$, then $\frac{h_{0} d_{0}^{-\alpha_{2}}}{\sum_{i=1}^{\infty} h_{i} d_{i}^{-\alpha_{2}}} \geq \frac{h_{0} d_{0}^{-\alpha_{1}}}{\sum_{i=1}^{\infty} h_{i} d_{i}^{-\alpha_{1}}}$, i.e., SINR increases with $\alpha$, which holds for any BS deployment and any user. Thus, Lemma 3 is proven.
Then, with Lemma 3, Theorem 3 is proven as follows:

Proof: According to Lemma 3, $\forall \alpha \in(2,4]$,

$$
\operatorname{Pr}(\operatorname{SINR} \geq T) \leq \frac{1}{1+\sqrt{T} \arcsin \left(\sqrt{\frac{T}{1+T}}\right)},
$$

which holds just by letting $\alpha=4$. Due to $\arcsin (x) \geq x$ $(x \in[0,1])$, we have,

$$
\operatorname{Pr}(\operatorname{SINR} \geq T) \leq \frac{\sqrt{1+T}}{\sqrt{1+T}+T}
$$

Let $T=2^{(n+1) \frac{u}{W}}-1$, which leads to the following:

$$
\begin{aligned}
\operatorname{Pr}\left(\operatorname{SINR} \geq 2^{(n+1) \frac{u}{W}}-1\right) & \leq \frac{2^{(n+1) \frac{u}{2 W}}}{2^{(n+1) \frac{u}{2 W}+2^{(n+1) \frac{u}{W}}-1}} \\
& \stackrel{(b)}{\leq} \frac{2^{(n+1) \frac{u}{2 W}}}{2 * 2^{(n+1) \frac{3 u}{4 W}}-1} \\
& =\frac{1}{2} 2^{-\frac{1}{4}(n+1) \frac{u}{W}} \sum_{m=0}^{\infty}\left(2^{-(n+1) \frac{3 u}{4 W}-1}\right)^{m},
\end{aligned}
$$

where (b) holds due to the mean inequality.

The rest is similar to the proof of Theorem 2, and is not shown in details.

\section{ACKNOWLEDGMENT}

The authors sincerely thank professor Jeffrey G. Andrews for his constructive comments and careful instruction on the presentation.

\section{REFERENCES}

[1] D. Cao, S. Zhou, and Z. Niu, "Optimal base station density for energyefficient heterogeneous cellular networks," in Proc. 2012 IEEE ICC.

[2] Ericsson, "Home NodeB output power," 3GPP TSG Working Group 4 meeting. Available: http://www.3gpp.org/ftp/tsg_ran/WG4_Radio/ TSGR4_43bis/Docs/.

[3] R. Kim, J. Kwak, and K. Etemad, "WiMAX femtocell: requirements, challeges and solutions," IEEE Commun. Mag., vol. 47, no. 9, 2009.

[4] V. Chandrasekhar and J. G. Andrews, "Uplink capacity and interference avoidance for two-tier femtocell networks," IEEE Trans. Wireless Commun., vol. 8, no. 7, pp. 3498-3509, 2009.

[5] H. Jo, C. Mun, J. Moon, and J. Yook, "Self-optimized coverage coordination in femtocell networks," IEEE Trans. Wireless Commun., vol. 8, no. 10, pp. 2977-2982, 2010.

[6] Tropos Networks, "Picocell mesh: bringing low-cost coverage, capacity and symmetry to mobile WiMAX," white paper. Available: http://www. tropos.com/pdf/whitepapers/tropos_whitepaper_picocellsforwimax.pdf. 
[7] Qualcomm Inc., "LTE advanced: heterogeneous networks," white paper, Jan. 2011. Available: http://www.qualcomm.com/documents/ lte-advanced-heterogeneous-networks-0.

[8] A. Damnjanovic, J. Montojo, Y. Wei, T. Ji, T. Luo, M. Vajapeyam, T. Yoo, O. Song, and D. Malladi, "A survey on 3GPP heterogeneous networks," invited paper, IEEE Wireless Commun. Mag., vol. 18, no. 3, pp. 10-21, June 2011 .

[9] Available: http://it.icxo.com/htmlnews/2010/05/14/1383534.htm

[10] M. A. Marsan, L. Chiaraviglio, D. Ciullo, and M. Meo, "Optimal energy saving in cellular access networks," in Proc. 2009 GreenComm ICC.

[11] S. Zhou, J. Gong, Z. Yang, Z. Niu, and P. Yang, "Green mobile access network with dynamic base station energy saving," in Proc. 2009 ACM MobiCom, poster.

[12] Z. Niu, Y. Wu, J. Gong, and Z. Yang, "Cell zooming for cost-efficient green cellular networks," IEEE Commun. Mag., vol. 48, no. 11, pp. 74-79, Nov. 2010.

[13] F. Richter, A. J. Febske, and G. P. Fettweis, "Energy efficiency aspects of base station deployment strategies in cellular networks," in Proc. 2009 VTC - Fall.

[14] B. Badic, T. O'Farrel, P. Loskot, and J. He, "Energy efficiency radio access architectures for green radio: large versus small cell size deployment," in Proc. 2009 VTC - Fall.

[15] Y. Chen, S. Zhang, and S. Xu, "Characterizing energy efficiency and deployment efficiency relations for green architecture design," in Proc. 2010 ICC Commun. Workshop.

[16] W. Wang and G. Shen, "Energy efficiency of heterogeneous cellular network," in Proc. 2010 VTC - Fall.

[17] K. Tutschku, N. Gerlich, and P. Tran-Gia, "An integrated approach to cellular network planning," in Proc. 1996 International Netw. Planning Symp.-Netw.

[18] N. Weicker, G. Szabo, K. Weicker, and P. Widmayer, "Evolutionary multiobjective optimization for base station transmitter placement with frequency assignment," IEEE Trans. Evolutionary Computation, vol. 7, no. 2, pp. 189-203, Apr. 2003.

[19] B. Lin, M. Mehrjoo, P. H. Ho, L. L. Xie, and X. Shen, "Capacity enhancement with relay station placement in wireless cooperative networks," in Proc. 2009 IEEE WCNC.

[20] E. Amaldi, A. Capone, and F. Malucelli, "Planning UMTS base station location: optimization models with power control and algorithms," IEEE Trans. Wireless Commun., vol. 2, no. 4, pp. 939-952, 2003

[21] E. Amaldi, A. Capone, and F. Malucelli, "Radio planning and coverage optimization of 3G cellular networks," Wireless Netw., vol. 14, no. 4 pp. 435-447, Aug. 2008.

[22] A. So and B. Liang, "A Lagrangian approach for the optimal placement of wireless relay nodes in wireless local area netowrks," in Proc. 2006 Int. IFIP-TC6 Netw. Conf., pp. 160-172.

[23] S. Hanly and R. Mathar, "On the optimal base-station density for CDMA cellular networks," IEEE. Trans. Commun., vol. 50, no. 8, pp. 1274 1281, Aug. 2002.

[24] D. Stoyan, W. Kendall, and J. Mecke, Stochastic Geometry and Its Applications, 2nd ed. John Wiley and Sons, 1996.

[25] M. Haenggi, J. G. Andrews, F. Baccelli, O. Dousse, and M. Franceschetti, "Stochastic geometry and random graphs for the analysis and design of wireless networks," invited paper, IEEE J. Sel. Areas Commun., vol. 27, no. 7, pp. 1029-1046, Sept. 2009.

[26] J. G. Andrews, F. Baccelli, and R. K. Ganti, "A tractable approach to coverage and rate in cellular networks," IEEE Trans. Commun., vol. 59, no. 11, pp. 3122-3134, Nov. 2011.

[27] H. S. Dhillon, R. K. Ganti, F. Baccelli, and J. G. Andrews, "Modeling and analysis of $K$-tier downlink heterogeneous cellular networks," IEEE J. Sel. Areas Commun., vol. 30, no. 3, pp. 550-560, Apr. 2012.

[28] M. Penrose, Random Geometric Graphs. Oxford Studies in Probability, Oxford University Press, 2003.

[29] E. Pineda and V. Garrodo, "Domain size distribution in a Poisson Voronoi nucleation and growth transformation," Physical Rev. E., vol. 75, no. 4, 2007. Available: http://pre.aps.org/abstract/PRE/v75/i4/ e040107.

[30] E. Pineda and D. Crespo, "Temporal evolution of the domain structure in a Poisson-Voronoi nucleation and growth transformation: results for one and three dimensions," Physical Rev. E., vol. 78, no. 2, 2008. Available: http://pre.aps.org/abstract/PRE/v78/i2/e021110.

[31] B. Rengarajan, G. Rizzo, and M. A. Marsan, "Bounds on QoSconstrained energy savings in cellular access networks with sleep modes," in Proc. 2011 ITC, pp. 47-54.
[32] C. J. Schrijver, H. J. Hagenaar, and A. M. Title, "On the patterns of the solar granulation and supergranulation," Astrophysical J., vol. 475, pp. 328-337, Jan. 1997

[33] R. N. Pupala, L. J. Greenstein, and D. G. Daut, "Throughput analysis of interference-limited MIMO-based cellular systems," IEEE Trans. Wireless Commun., vol. 9, no. 6, pp. 1946-1951, June 2009.

[34] Available: http://www.umtsworld.com/technology/wcdma.htm

[35] S. Singh, H. S. Dhillon, and J. G. Andrews, "Offloading in heterogeneous networks: modeling, analysis and design insights," submitted to IEEE Trans. Wireless Commun. Available: http://arxiv.org/abs/1208. $1977 \mathrm{v} 1$.

[36] EARTH project, "Definition and parameterization of reference systems and scenarios," June 2010. Available: https://www.ict-earth.eu/ publications/deliverables/deliverables.html.

[37] EARTH project, "Energy efficiency analysis of the reference systems, areas of improvements and target breakdown," Dec. 2010. Available: https://www.ict-earth.eu/publications/deliverables/deliverables.html.

[38] S. Boiardi, "Radio planning of energy-aware cellular networks," Ph.D thesis, Politecnico di Milano, 2010. Available: https://www.politesi. polimi.it/bitstream/10589/12083/1/thesis.pdf.

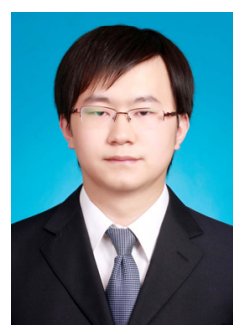

Dongxu Cao (S'10) received his B.S. degree in Electronic Engineering from Tsinghua University, China, in 2011. He is currently a Ph.D. student in Electronic Engineering Department at Tsinghua University. His research interests include network planning and energy-efficient operation for heterogeneous cellular networks.

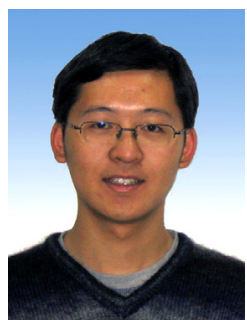

Sheng Zhou (S'06, M'12) received his B.S. and $\mathrm{Ph} . \mathrm{D}$. degrees in Electronic Engineering from Tsinghua University, China, in 2005 and 2011, respectively. From 2011 to 2013, he was a postdoctoral scholar in Electronic Engineering Department at Tsinghua University, where he is currently an assistant professor. From January to June 2010, he was a visiting student at Wireless System Lab, Electrical Engineering Department, Stanford University, CA, USA. His research interests include cross-layer design for multiple antenna systems, cooperative transmission in cellular systems, and green wireless cellular communications.

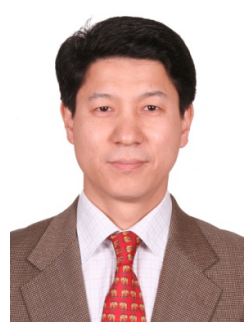

Zhisheng Niu (M'98, SM'99, F'12) graduated from Northern Jiaotong University (currently Beijing Jiaotong University), Beijing, China, in 1985, and got his M.E. and D.E. degrees from Toyohashi University of Technology, Toyohashi, Japan, in 1989 and 1992, respectively. After spending two years at Fujitsu Laboratories Ltd., Kawasaki, Japan, he joined with Tsinghua University, Beijing, China, in 1994, where he is now a professor at the Department of Electronic Engineering, deputy dean of the School of Information Science and Technology, and director of Tsinghua-Hitachi Joint Lab on Environmental Harmonious ICT. He is also a guest chair professor of Shandong University. His major research interests include queueing theory, traffic engineering, mobile Internet, radio resource management of wireless networks, and green communication and networks.

Dr. Niu has been an active volunteer for various academic societies, including Director for Conference Publications (2010-11) and Director for Asia-Pacific Board (2008-09) of IEEE Communication Society, Membership Development Coordinator (2009-10) of IEEE Region 10, Councilor of IEICEJapan (2009-11), and council member of Chinese Institute of Electronics (2006-11). He is now a distinguished lecturer (2012-13) of IEEE Communication Society, standing committee member of both Communication Science and Technology Committee under the Ministry of Industry and Information Technology of China and Chinese Institute of Communications (CIC), vice chair of the Information and Communication Network Committee of the Chinese Institute of Communications (CIC), editor of IEEE Wireless Communication Magazine, and associate editor-in-chief of IEEE/CIC joint publication China Communications. He received the Outstanding Young Researcher Award from Natural Science Foundation of China in 2009. He is now a fellow of IEEE and IEICE. 